\title{
Infectious Disease Pharmaceutical Care: The Role of the Clinical Pharmacists to Improve Clinical Outcomes 2017
}

\section{Luisetto $\mathbf{M}^{\star}$}

European Specialist in Laboratory Medicine, Hospital Pharmacist's Manager, Italy

*Corresponding author: Luisetto M, European Specialist Laboratory Medicine, Hospital Pharmacist Manager, 29121, Italy, Tel: 393402479620; E-mail: maurolu65@gmail.com

Received date: June 12, 2017; Accepted date: June 16, 2017; Published date: June 23, 2017

Copyright: ( 2017 Luisetto M. This is an open-access article distributed under the terms of the Creative Commons Attribution License, which permits unrestricted use, distribution, and reproduction in any medium, provided the original author and source are credited.

\begin{abstract}
Today more than past we need to improve clinical outcomes in the management of infectious disease related to patient in critical conditions (septic shock, sepsis, MOF, invasive fungal infections, HIV, peritonitis, meningitis, endocarditis, osteomyelitis, pneumonia, severe UTI, proteases infections and other). The same way we can see the high efficacy of some new drugs in eradicate severe infectious (as CEPAT), saving cost for transplant. This condition needs today an active role and permanent presence of clinical pharmacist in medical team because these therapies highly involved in patient's health.
\end{abstract}

The objective of this work is to analyze the role of clinical pharmacist in infectious disease medical team observing some in our opinion relevant publication and the positive effect on clinical outcomes. Infectious disease needs today new management systems to contain the increase of antimicrobial resistance in an era of few new and innovative drug discovered by pharmaceutical industries.

Clinical pharmacist can give to the physicians a great help in this field to improve clinical outcomes, reducing toxicity, containing costs but also to preserve the antimicrobial potential of the antimicrobials (as safe-life drugs). These are only some of the many reason to apply the clinical pharmacist competence in patient medical team.

Keywords: Infectious disease; Pharmaceutical care; Clinical pharmacy; Clinical outcomes; Pharmaco-economy; Health care management

\section{Introduction}

Some infectious disease aspects must be considered in priority way:

- Few new antimicrobial molecules have been produced in last time (new synthetic lipo-glicopeptide like Dalbavancin and Cepaholosporin of $4^{\text {th }}$ generation with penicillin inhibitors, or associated to avibactam, second generation of oxazolidinon molecules as tedizolid few other).

- Wide spectrum antimicrobial ABUSE (contributes to give diffusion to MDR)

- MDR is rapidly expanding (and old disease are diffused also in advanced countries due also to more easy travel from tropical climate, migration) as TBC.

- The need of re-introduction in therapy of old antimicrobials (as colimicin or parenteral fosfomicin) to be used in resistance patterns.

- Abuse of some crucial antimicrobials out of hospital in outpatient's (increase resistances).

- The migration of great number of population in different world area contributes to the diffusion of infectious disease as well as the easy travel way of tourists from endemic area. (Ebola, H1N1 virus, Zika or TBC, MDR are example of recent health problem we have today associated to few new antimicrobial and antiviral molecules associated with high level of resistance).
- The total cost for antimicrobial therapy constantly expands.

These are only few reasons to use antimicrobial drugs in better conditions. We need to apply the best strategies to use the drugs we have today available. Infectious disease management systems include: Physicians, clinical pharmacist with expertise in antimicrobial stewardship added to microbiologist, epidemiologist, statistical data analysts and nurse can be the right team. But also other professionals as surgeons, ICU healthcare professional, budget control office, risk analysis and general managers, infectious disease committee and other relevant service). The right decision making activity and operative actions gives more efficacies in global clinical response and cost control. So we can say that multi-disciplinarily is the right way of working. But a rational use of antimicrobials need in special way of clinical pharmacists expertize as they are considered internationally as the drugs expert for excellence (due to the pharmaceutical, pharmacological, toxicological, metabolism, kinetics deep knowledge). Some measure can help in the global management such as:

- Limitation antimicrobial drug use systems central and local rules.

- Health ministry and government policy.

- Preventive measure, isolation measure.

- Single patient prescription with motivation, the clinical pharmacist must verify the prescription (accepting or not).

- Antimicrobial stewardship.

- Antimicrobial drug use control, Pharmaco-economy.

- Local drug hospital formulary committee that include the antimicrobial drugs (to limit the drug use).

- EBM based activity (efficacy verified): Protocols, procedure, guideline, ministerial rules. 
- Clinical pharmacist counselling and other tools available towards healthcare professionals, patient (in example TBC; HIV) or care givers and in specific local educational programs (preventive activity).

- Antibiograms.

- Resistance pattern.

- Antiseptic and disinfectant policy.

- Clinical audit in the different hospital wards make possible to contribute to monitoring of misuse of antimicrobial drugs.

- Preventive measure: Antimicrobial prophylaxis before surgery or therapy, after emergency surgery (trauma), vaccination strategies, antimicrobial prophylaxis before travelling, isolation measure, sterile procedure and other that can contain the diffusion of the disease.

The infectious disease team must acts as a coordinating center to monitoring the full process: A rapid communication system between the many professionals involved (in some in severe cases in rapid way) makes the difference in critical conditions.

The top of organization (chief medical office) and since to the bottom level since single healthcare professional are heavily involved (for example; the infectious disease hospital committee, local hygiene office acts in this way). Other service interested is for example: Central and clinical pharmacy, microbiology lab, pathology lab, imaging department. Before analyzing some in our opinion relevant bibliography we observe some aspect of infectious disease therapy.

Many bacteria, viruses, fungi, protozoa, helminths and other parasites are involved in severe human infectious disease. This pathology can be acute (also with high mortality rate) is bacterial meningitis or chronic such as diabetes food (TBC in example) and frequently associated with high healthcare costs. Critically patient can be geriatric patient, transplanted or with cardiovascular disease, pulmonary, diabetics, nephrological but also with many other severe conditions. This work is involved in priority way the bacteria infectious disease but some consideration can be useful also in other kind of infectious disease. The same is not our intention to give details about systematic therapy but some general useful information. Clinical pharmacist in infectious disease equipment must consider patients conditions, apparatus and organ status, polypathology microbes characteristics, infectious way of transmission, antimicrobials used, environment characteristics, resistance profile and other relevant aspect to be necessary evaluated. In this pathologies the characteristics of the microbes, added to the patient condition in a specific environment can gives severe conditions level.

\section{Patient data and condition}

Following points must be considered for patient data condition:

- Polypatholgy, severe disease, elderly, immuno-depressed, transplanted and other relevant conditions (many risk factor easy gives critical conditions).

- Medical and pharmaceutical anamnesis and history: Profession, geographic provenience, travel, food behaviours lifestyle, sexual habits: (useful data for isolation and other measure) diagnosis and treatment.

- Risk factors.

- Sign and symptoms, Med lab, imaging and clinical data, fever evolution, lymph nodes, diarrhoea, cough, dermatitis and other.

- Clinical and instrumental diagnosis.
- Etiology, diagnosis.

- Acute or chronic infectious disease definition.

- Therapy (politherapy) other pharmacological therapy.

- Transfusion states, haemo-derivatives used history. Immunization history, vaccination.

- Comorbidity: HIV, Oncologic disease, Neprologic disease. Cardiovascular disease, pulmonary disease, Epathic disease and other.

- Physiological status: Pediatric, geriatric other, particular condition (pregnancy breast feeding and others).

- Organ and systems function level (useful in example in some kind of antimicrobial toxicity): Renal, liver, cardiac, bone marrow failure and other.

- Severe hill patients, ICU (for eg. MOF, sepsy, invasive fungal disease meningitis, peritonitis, pneumonia).

- Smokes, alcohol, drugs use (smoke gives more pulmonary infectious and cardiovascular disease and alcohol gives hepatic and pancreatic and gastrointestinal problems).

- Other therapies that can influence the therapy (cyclosporine, cortisone, onco-chemioteapic, mabs).

- Profilaxes procedure (yes or not).

- Surgery.

- Addictions conditions: Heroin and other.

- Oncologic therapy.

- Bites (animals, ticks).

- Trauma (simply, complex).

- Pregnancy condition (molecules permitted or not).

\section{Diagnostics}

In these cases is generally considered:

- Kind of infections: Viral, bacteria, protozoa, helminthes or other.

- Clinical presentation: Laboratoristic, imaging and instrumental data (serologic, microbiologic, cultures, antibiograms, immunology, molecular biology).

- Indirect or direct methods: Immunological, antigen (virus).

- Imaging: RX traditional and other techniques in ex-nuclear medicine. Infectious and chemo-sensibility are verified and confirmed by lab test.

- Sign and symptoms: In meningitis typical at position, peritonitis, exanthema, neutrophilia, fever, vomiting, diarrhoea, coma, lymph nodes, dehydration, analytical position, and many other examples.

\section{Environment characteristics}

These conditions can influence diffusion of the disease and natural history of the pathology. Few of such conditions are:

- Travel history: Tropical, subtropical (TBC, malaria other).

- Local ecology (microbe's population and resistances).

- Migrations.

- Hospital setting, residency, homecare, community.

- Hygienic conditions (water).

- Food conditions (safety, malnutrition conditions).

- Climate for eg. Tropical climate. 


\section{Bacterium properties}

Intra or extracellular, Host, transmission modality, Sporogenic or asporogenic bacteria, Capsulated or not capsulated bacteria, Toxins (endotoxins and exotoxins) adhesives, opportunistic or not, virulence, pathogenicity, gram positive and gram negative, atypical, genetic resistance profile (HIV), biofilm characteristics etc. are few bacterium property.

\section{Infectious characteristics}

- Bacterial charge.

- Diffusion characteristics: Endemic, pandemic, epidemic.

- Way of transmission: Direct contact, sexual, by contiguity, skin, oro-pharynx and gastro-intestinal, oro-faecal, respiratory, CVC trauma, surgery, etc.

- Local or systemic disease.

- Simply or complicated.

- Acute or chronic.

- Focus diffusion: From intravascular or extravascular.

- Aerobic, anaerobic, mixed.

- Food toxin infections, toxins (exotoxins and endotoxins).

- Recidives

- Immune carrier.

\section{Hosts}

Some example of hosts can be, Yarsinia pestis (Mouse), $P$. falciparum (Anopheles), Brucella melitensis, and others can control and reduce disease diffusion.

\section{Antimicrobials}

- Drug classes: Antibacterial, antivirus, antifungal, antiprotozoal and other classes.

- Spectrum: Gram positive, Gram negative, Atypical, Anaerobic large, tight spectrum (ampicillin and piperacillin wide spectrum).

- Kinetics and dynamics: Nephrotoxicity, ototoxicity, other toxicity (bone marrow-CAF).

- Bacteriostatic or bactericide: (Severe infectious diseases need bactericide).

- Action: Concentration or time dependence.

- Target: Ribosome, cell wall, cellular membrane DNA, RNA, metabolic enzyme toxicity (selectivity, bacterium vs. human).

- Ways of sub-administration: Parenteral or not.

- PAE: Post antibiotic effect.

- Tissue diffusion and penetration (sanctuaries), intracellular, biofilm.

- Drug Generation: In example cephalosporin of $1,2,3,4$ generations (different spectrum of activity).

- Enzymatic inhibitors: in ex-beta lattamase inhibit. To reduce bacterial inactivation.

- Protected penicillin (to prevent resistance).

- Indications registered, off label, contraindications.

- Dialysis properties (dialyzable or not).

- Activities in bacteria growth phases (with penicillin) or in quiescent phases (with aminoglycoside).

- Biofilm penetration, etc.

\section{Antibiograms}

EUCAST: Clinical breakpoint and epidemiological cut off.

a) Wild type (He has not acquired resistance)

b) No wild type

MIC (Minimum Inhibitory concentration)

Antimicrobials Sensibility (R, S, I)

\section{Pharmaceutical properties}

- Pharmaceutical form: Parenteral, oral, topic.

- Preparation and stability, solvent.

- Storage condition.

- Expiration data.

- Lipophilic idrophilic.

- Liposomal.

- Retarded release (penicillin depot).

\section{Immunomodulants, citochines (added to the therapy)}

- Interferon

- Filgrastim

- Other immune-modulators

\section{Uses: Some examples}

- MRSA: Oxacilin, Vancomicin, Teicoplanin

- Clostridium difficile: Metronidazole vancomicin

- Gram positive MDR: Daptomicin

- Gram negative MDR: Cephalosporin $3^{\text {rd }}$ generation (more active than 2 generation)

- Pseudomonas areuginosa: Cefepima wide spectrum.

- Enterobacter species: Meropenem, imipenem, aminoglycoside.

- Carbapenemic gram negative anaerobe multi-resistant: Tetracycline wide spectrum.

- Actinobacter. Imipenem, aminoglycoside.

- H1N1: Oseltamivir (treatment and prophylaxis).

- K. pneumonia: Cephalosporin, aminoglycoside.

- Clindamycin for diabetic food, osteomyelitis, peritonitis (anaerobic).

- Tycacil for complicated infection abdominal tissue.

- Antipseudomonas piperacillina: Aminoglycoside+colistin.

- Penic antipseudomonas piperacillina: Ciprofloxacin.

- Linezolid gram+multiresistant MRSA VISA, VRSA, VRE.

- Pseudomonas (pulmonary infection), UTI: Fluorochin.

- Lincosamide clindamycin: Gram positive anaerobe, problem clostridium diff., Nitrofurantoin for some urinary infections.

- Gram positive MDR: Glicopep tric vaccine.

- Pneumocystis carinii: Trimethoprim-sulfamate.

\section{Contraindications: Some examples}

Tetracycline not in pediatric, parenteral penicillin not in allergic patients, hyper sensibility, penicillin, renal failure (aminoglycoside), hepatic failure, pregnancy, ontological toxicity (aminoglycoside), tetracycline not in pediatric patient, parenteral penicillin not in allergic, bypass, etc. 


\section{Interactions: Some examples}

Inducer and inhibitors metabolism, GI adsorption, enteroepathic recircle, polipharmacy, elimination phases, organ failure (empathic, renal).

\section{Side effects and toxicity}

Some examples of local or systemic reactions are:

- Vitamin K reduced synthesis, GI flora modification, diarrhoea.

- Allergy: Penicillin (even fatal event anaphylactic shock).

- QT modify: Macrolides.

- Hepatitis: tetracycline.

- Nephrotoxicity: Aminoglycoside (increase if added other nephrotoxic drugs), Vanco, teico, amphotericin B high dose, Ototoxicity,

- Hematological disorder: Trimethoprim, Steven-Johnson syndrome.

- Neurological effects: Chinolon, cutaneous erythema.

- Vancomicin: Red man syndrome, Nephrotoxicity aminoglycosides, hepatotoxicity oxacillin, Rifampicin, tetracycline.

- Ethambutol: Visual problems.

- Pseudomembranous colitis: (Clostridium difficile): Clindamicin cephalosporin, Fluorochinolon.

- Chloramphenicol: Grey baby syndrome.

- Isoniazid: Peripheral neuropathy.

- Tetracycline: Photo-sensibilization, teeth colored in pediatric patients.

- Haemorrhagic gastroenteritis: Amphotericin B.

- Pancreatitis.

- Bone marrow toxicity: Chloramphenicol.

- Massive bacterium killing syndromes with cytokine release (TNF)

- Electrolytes overload (CA, MG) with $K$. penicillin) for eg. penicillin high doses, curaric effect (by Mgs alts aminoglycosides).

- Cardiotoxiticy (K. penicillin).

- Platelet paenia (some beta lactamic) and coagulopathies.

- Neurotoxicity (otology ex aminoglycosides), encephalopathies.

- Anti-androgen effect, mineral corticoid effect, ketoconaozl metabolic acidosis (in renal failure or neonates) some antimicrobials modify of the natural bacteria flora. (Pseudomembranous colitis by res. Clostridium difficile in example using clindamicina and some penicillin biliary excreted as ceftriaxone, ureidopenicillin) enterococcus after cephalosporin $3 \mathrm{G}$ use MRSA after use of imipenem or celphalosporin $3^{\text {rd }}$ generation.

- Candida after wide spectrum antimicrobial use.

\section{Monitoring-TDM}

Monitoring can be in clinical or laboratory way. TDM therapeutic drug monitoring toxicological lab. Used as instrument to drive the therapy in some glycopeptide and aminoglycoside therapy preventing toxicity. Therapeutic efficacy and viraemia (HIV). Clinical conditions: Fever, lymph nodes, white blood cells, IG, Underdosage, Overdosage, Duration of therapy, compliances.

\section{Preventive measures}

Not overuse of antimicrobials in zootecnia, Hospital infectious disease, preventive office, hygienic measures, Notify Warning to health local office about diffusive infectious disease. Epidemiology, infection prevention and control of infections, water source disinfections and safe use disinfectants rotation vaccine prophylaxis (papillomavirus), IG therapy, isolation measures, sterilization procedure, washing hands procedure (surgery and other), antimicrobials surgery prophylaxis (cephazolin), Nutritional measure (vitamins, proteins) in ex Africa pop., medical devices single use, travel prophylaxis (malaria), Information activity, Formative activity, dpi use (EBOLA, HIV, hepatitis, etc), prophylaxis of endocarditis (before dentistry procedure), prophylaxis haemophilic: Riphamicin.

\section{Compliance problems}

This can high influence the efficacy of some kind of therapy. TBC, HIV and other long-time therapy. The same economic conditions are involved in the availability of drugs. Age, elderly conditions make more difficult to have a correct compliance due to cognitive failure.

\section{Non drug therapy some examples}

UTI preventive IDRO-THERAPY, not use water in opened bottle in countries with endemic infectious disease, sexual behaviour, not smoke to prevent pneumonia, nutrition level, right dosage of vitamins and proteins healthy environment, etc.

\section{Kinetics}

Some kinetics is related to antimicrobial therapy. Some examples are $\mathrm{t}^{1 / 2}$, bypass, placental pass, lipophilic-idrophilic balance, molecular weight, AUC, clearance, first pass effect, steady state, kinetic absorption-elimination rifampicin biliary elimination (for TCB, MRSA, osteomyelitis meningitis, endocarditis and legionella), dialysis properties (amphotericin not dialyzable), Idrofilic: Beta calamine, glycopeptide, aminoglycoside: limited to VD. Low passive diffusion in cell eukaryotes. Inoculations on intracellular bacteria eliminate renal fever unmodified. Acid or bases (trimethoprim) properties. Lipophilic: Macrolides florochinol, tetracycline, CAF, Rifampin. Linezolid. Wide VD: Diffusion in cell membrane of eukaryotes active in intracellular infectious, hepatic elimination.

\section{Therapy}

Dosage, frequency, posology and days of treatment. Right drugs, right patient, right time of sub ministration, right posology. Approach: empiric or focused. Patient's weight to age (to choose the posology), ways: IV, IM, OS, topical. Time or concentration depend use strategy, Sub ministration: Oral or parenteral, load doses, duration of therapy (in example form 3-7 days to 6 month or more), switch with OS (to reduce costs), less complexity in management mono therapy or association's therapy. For example, empiric therapy in ICU, severe disease MDR, to cover wide spectrum of activity since from first time.

Down shift therapy: De-escalation of empiric choice related to lab test of antimicrobial sensibility (migrated), this reduce the total costs and MDR development. In example reduced the antimicrobials used in a patient therapy. (for example; Starting from 3 molecules to only one) when clinically permitted. Posologic adjustement is related to patient conditions, kind of infections, dynamic and kinetics. Parenteral/oral subministration shift when clinically permitted reduce days of recovery and costs. (Following guidelines and hospital procedure). Antimicrobials drugs choice should be first, second, third choice depending upon clinical or microbiological). 
Dosage could be charge dose, maintenance, daily dosage. TDM, biochemistry and clinical lab data is used for monitoring of antimicrobial therapy. Duration of therapy is related to infectious patient, pathogen and infectious characteristics. Surgical options for example, in asceccusal or protesisis infections optimize posology (time or concentration dependent) to prevent new MDR safeguard activity. Concentration dependent molecules such as mono subministration reduce the renal accumulation. Antimicrobial concentration on infectious site: If concentration is $>$ MIC probably we can have more eradication and if it is $<$ MIC, inefficacy in eradications. for example, chronic prostatis, osteomyelitis. Antibiograms use (MIC, MIB) to drive therapy. Migrated or empiric therapy is used when possible. Better to use migrated therapy to prevent MDR diffusion). In difficult etiological diagnosis, initially we use wide spectrum therapy to cover the most frequent microbes for ex in pneumonia. In this situation if too much time to wait for lab test or cultural results and therapy is not started early we can have critical consequences. Set aside some antimicrobial in hospital setting and not in out of this settings make possible to prevent diffusion of resistances.

TDM used tin posology adjustment phases. In some classes of antimicrobials, nomograms use in renal failure for example, vancomicin, amikacin making possible adjusting dosage or frequency of subministration reducing the organ toxicity. TDM at the steady state or after posology modify. Valley or peak plasma level and Sequential antimicrobial therapy are another therapy. De-escalation therapy is a useful strategy when microbiological data gives information about etiology, antimicrobial sensitivity spectrum and reduce costs and resistance. It is useful in having rapid concentration of antimicrobial in bloods and tissue (then continuous infusion or oral sub). As per kinetics is concerned it is easy for adsorption, metabolism, elimination.

It can be administered through IV, IM, oral, topic and aerosols. Switch therapies IV and oral therapy, when possible (stabile patient) will give reductions in costs and reduction in nurse time due to parenteral drugs management (with the same clinical outcomes). Antibiograms evaluation is used to drive the therapy. Failure therapy has its significance in clinical, immunologic and virology (HIV). Wound can be classified as clean, contaminate, dirty and trauma contaminated. Association of therapy is used when are synergic, increase spectrum of actions or mixed infectious or to reduce toxicity of co-sub antimicrobials. Associate bactericide and bacteriostatic is not used at the same time because this would reduce their activity.

\section{Antimicrobial resistance: Some examples}

Inappropriate antimicrobial drugs use, can induce MDR multidrug resistance for example in case of Clostridium difficile diarrhoea. Resistances can be naturally or acquired (chromosomal or extrachromosomal, spontaneous mutation, plasmid mediated). It's a resistance gene exchange between microbes, mutation and selective process. Chromosomal depend by bacteria chromosome (inside) and extra-chromosomal depends on extracellular factors (outside). Cross resistances (between drugs classes) must to be considered for drug therapy. MDR biochemical mechanism consists of following phases:

- Inactivation of antimicrobials.

- Modify target of antimicrobial activity, new target expression.

- Modified permeability.

- Reduce target affinity link (PBP).

- Inactivation (enzyme produced by microbes for example beta lactamase) KPC carbapenemase.
- Reduced intake of antimicrobial in intracellular site.

- Producing transmembranal extruder of antimicrobials.

MDR is multi-resistant to one or more antibiotic classes and sensitive to a few molecules. They are responsible for high mortality rates and costs and have limited therapeutic options. Attention to contamination routes is host, hands instrumentation etc.

\section{Resistances: Few examples}

- Few examples of resistances are as follows:

- Beta lactamase resistances: ESBL, KPC, AMPC, OXA, MBL

- MRSA: Methicillin resistance.

- Vancomycin resistance: VRSA (Vancomycin Resistance S. aureus)

- VRE enterococci are vancomycin resistance.

- MRSE, VISA, STMR, ESBL (Extended-spectrum beta-lactamases) are wide spectrum beta lactamase producers

- Resistance MLB macrolide lincosamide.

- KPC-MBL carbapenemase.

- MDR (Multiple Drug Resistances).

Bacteria are high involved in MDR. Few of them are as follows:

- Pseudomonas aeruginosa MDR are resistant to aminoglycoside, chinolonic, Cephalosporin $3^{\text {rd }}$ generation, carbapenem resitences (no monotherapy)

- Actinobacter baumanii and Stenotrophomonas maltophilia are resistant to Penicillin (AMP, PIP, TIC), Penicillin+beta lactamase inhibit, wide spectrum cephalosporin, monobattam, carbapenem, aminoglycoside reduced uptake, Trimetoprim, Fosfomicin. Acquired resistance is induced by chinolons (effluss pump), trimethoprim sulfametossazole metallo-beta-lattamase producer.

- $S$. aureus penicillin and vancomicin resistance. Acinetobacter baumanii MDR: KPC $K$. pneumoniae, a bacterial enzyme can hydrolyze carbapenemic drugs and cefalosporinin $4^{\text {th }}$ generation. ESBL beta lattamasi wide spectrums active also towards cefotaxima, aztreonam etc

- Enterococcus (E. faecium vancomycin-glicopept teico responder).

- Enterobacteria resistant; A cephalosporin, chinolons and carbapeneme.

- Pneumococci penicillino resistance.

- Streptococci macrilde resistance.

- Meningococci pen resistance.

- TBC-MDR.

The wide use of large spectrum of antimicrobial increase diffusion of resistances as well as a misuse of antimicrobials. This create great diffusion of MDR and to know the local, regional, national, international situation about resistance level give great information useful in therapy management.

\section{Medical lab test results, imaging, instrumental data}

Med lab test, imaging, Eco-graft, TC and other techniques. Other examples includes

- Haematological tests: Complete blood count, leukocytosis, monocytosis in mononucleosis etc, neutrophilia in bacteria infectious, leucocyte formula

- Lombar puncture (do differentiate bacteria from virus infectious)

- Flogosis marker: VES, PCR, GB, Procalcitonin. 
- Cultural test: for UTI, haemo-culture, other cultural assay.

- PCR (TBC), HIV; viraemia.

- Immunologic state.

- Immunometric assay.

- Resistance phenotype (in example for HCV, HIV), and many other tests.

Sensibility of analytic methods gives information about the data we have obtained in order to correctly use it.

\section{Viral infectious}

Viruses are involved in critical infectious. HIV, HCV, CMV, hepatitis, Varciella zoster, herpes and many other disease (viral meningitis), H1N1, syncytial respiratory virus are some examples. Today we have to manage high costs for some antiviral drugs HCV (Sofosbuvir and other drugs) phenotype (4 for HCV). Drugs with different profile of actions (use immunotherapy or associations to reduce it) high efficacy in therapy (about $90 \%$ eradication in some cases). In other disease as HIV we can see high resistance profile, for e.g. HIV: high level mutation/transformation. So is crucial to monitoring of efficacy of the therapy (clinical, laboratoristic), evaluate toxicity. The association use (HIV, HCV) can give improving in clinical outcomes. In some cases the acute infectious with drugs is modified as chronic pathology. Modify of associations if reactivation of infectious or toxicity is commonly used (UIV). Compliance problems can reduce the compressive efficacy. In other cases is used classic therapy: Herpes acyclovir CMV ganciclovir, Respiratory syncytial ribavirin.

\section{Prions}

Creutzfeldt-Jakob disease, Spongiform encephalopathy.

\section{Mycology infections}

- Hematology and oncologic settings: Immuno-depressant condition, transplant conditions. Apergillus, Mucor, candida can give severe ill that associated to the patient conditions can results in fatal event.

- Drugs: must be considered nephroticity, efficacy and costs.

- Drugs classes: Amphotericin, Echinocandin, Voriconazole and other azole molecule.

- Target of actions of the different drug classes.

- Toxicity.

- Classified in topic or systemic.

Some examples are as follows: Invasive candida, amphot b+flucityosin fluconazole, Aspergillus invasive amphoterin $\mathrm{B}$, Criptocossus- amphoterin B, flucitosin, fluconazole

Resistances: Especially mucor, Fusarium

\section{Protozoa and parasitology}

Must be considered great healthcare world problem in some countries. For example malaria by $P$. falciparum (blood, tissue or emphatic phases) e.g. Tripanosiomiasis, toxoplasmosis, amoeba, Entamoeba histolytica, Cryptosporidium, Pneumocystis.

\section{Helmintes}

Some helmintes infections are diffused in the world. For example: Nematodes, Strongyloidiasis, Cestodes, Trematodes, Filariasis.

\section{Ectoparasites}

Scabies, Pediculosis topical treatments.

\section{Normative rules}

- Vaccination oblige programs.

- Notify serious infectious disease to local health office.

- Useful instrument to reduce antimicrobial misuse to reduce costs and resistances.

- Limitative administrative rules (motivate request uses).

- Prontuaries policy (national, regional, local, ward).

- Off label management.

- Clinical audit (best practice).

- Protocols and procedure (surgery antimicrobial prophylaxis).

- Informatics blocks systems.

- Budget control.

\section{Antimicrobial management pharmacoeconomy}

- Strategies for rational use and cost control:

- Vaccination programs.

- Enhance environmental and nutritional conditions.

- Antimicrobial stewardship programs (antimicrobial stewardship can reduce about $30 \%$ antimicrobial use, reducing resistances and improving clinical outcomes).

- Patient's prescription assessment.

- Participation to infectious disease team (clinicians, microbiologists, clinical pharmacist, statistics, epidemiologist, nurse).

- Improving clinical outcomes and reducing mortality rates.

- Reducing toxicity activity.

- Cycling-rotation of antimicrobials and disinfectants strategy.

- To reduce resistance diffusion statistical analysis.

- Cost analysis antimicrobial use (vs. efficacy), hospitalization days number economic analysis (antimicrobials, antivirals, antifungal, vaccines, sterile procedure costs).

- Use index (number of infections, antimicrobials use and posology.

- Costs, Percentage of resistance, MDR, antimicrobial sensitivity.

- Percentage migrated therapy vs. empirical.

- Duration of therapy (3-5-7 days, month).

- Oral/parenteral ratio.

- DDD by recovery days.

- Clinical data: Morbidity infectious related mortality.

- Re-hospitalizations.

- Healthcare costs (total and analytical by molecule, wards).

- Percentage of switch therapy, percentage of de-escalation therapy.

- Medical devices use disinfectants.

- Clinical pharmacist time use (Human resource management).

- Antimicrobial hospital use (local regional national international).

- Infectious disease hospital committee activity. 


\section{Strategies to improve clinical outcome reducing toxicity or costs}

Strategies to improve clinical outcome reducing toxicity or costs are:

- Health nation and local policy.

- Prophylaxis and isolation measure.

- Decontaminant measure (nasal mupricocin pommade for Staphylococcus aureus carriers).

- Sterile procedure.

- Surgical washing hand.

- Antimicrobial stewardship: Priority in wards with huge antimicrobials use (to reduce morbility and mortality ratio).

- Protected penicillin use: To reduce enzymatic inactivation (Piperacillin-tazobactam, tazocin and $S$. aureus infectious).

- New formulation: Liposomal amphotericin to reduce nephrotoxicity.

- Use of association: TBC, HIV etc. to reduce resistances.

- Choosing therapy according to the patient pathology: Renal failure or hepatic failure.

- ICT USE, informatics prescription process, traceability: microbiological data, antibiograms.

- Rapid lab responses in high risk situation, rapid alert systems of infectious and resistances. (Isolation measure, IG prophylaxes). A rapid system of communication in hospital makes possible rapid measure (with isolation and other).

- Stop therapy when possible if not necessary.

- Posologic adjustment: Charged doses, maintenance, switch parenteral and oral.

- Procedure, protocols guideline, EMB based.

- Resistances, local situation.

- Policy strategies: rotation of antimicrobials classes, disinfectants.

- Washing hands (febrile neutropenia oncology), sterile procedure (surgery, CVC).

- Synergic associations use: for eg. aminoglycoside penicillin.

- Triple therapy in HIV, TBC.

- Associations dangers: Between nephrotoxic drugs.

- Renal failure: Strategies in example reduce dosage, time between sub., use non-nephrotoxicity antimicrobial if possible.

- Antimicrobial to be used only if: Prophylaxis is need or infectious present, right drugs indication of use (registered or off label).

- Drugs limitation use strategy (slectionates settings, ICU, surgery, transplants and infectious disease ward).

- Right dosage, time correct, right frequency and right way of submitting charge doses.

- Right concentration in infectious site (or blood) monitoring.

- Rigorous Stop therapy when possible (clinical condition).

- Co-subministration of drugs only if really necessary (cot cross reactive possibly).

- Use of narrow spectrum antimicrobials when possible.

- Accreditation systems (structure, professionals, procedure).

- Time of subministration in prophylaxis respect and verify updating activity to healthcare professional, patient and caregiver, task force (Ebola), Briefing focus on activity.

- Team working.

- Removing activity to implementation of procedure (cultural).
- New drug, research, Industries strategy: Discovering new molecules.

- Presence of adequate systems to have epidemiologic data. (Infectious, antimicrobial use, resistance).

- Low knowledge and skills, generic drugs use, buying strategy, drugs price assessment national policy, regional-local buying system.

\section{Material and Methods}

In this review work we use an observational approach observing in our opinion some relevant literature about pharmaceutical care in infectious disease management and the effect on clinical and economic outcomes.

\section{Results}

We have found in this literature:

Oman wrote "To characterize the pharmaceutical care provided by a clinical pharmacists working in a tertiary health care institute, where the extent to which the pharmacist contributes to changes in prescribing patterns use of medications, and patient knowledge was described.

A retrospective single cohort study design was used. Qualitative and quantitative evaluation of a documented pharmaceutical care plan was undertaken. Electronic pharmaceutical care descriptor (Microsoft Access database) was used for analysis. A total of 167 patients (mean age of 53 years, $70 \%$ male) from a Medical Health Centre in a tertiary hospital where a clinical pharmacist had provided a medication review. The study patients were those who had either been admitted to cardiology or infectious disease wards.

There were 291 pharmaceutical care issues (PCIs; mean per patient [2]) comprising of $67 \%(\mathrm{n}=194)$ relating to treatment monitoring and $33 \%(n=97)$ relating to treatment changes, representing a total of 291 drug therapy problems (DTPs; mean per patient [2]). The resolution rate of DTPs was $70 \%$, where $61 \%$ of recommended changes and $75 \%$ of recommended monitoring were implemented.

The clinical pharmacist successfully addressed most PCIs while attending ward rounds, reviewing in-patient prescriptions and counseling discharged patients. The electronic pharmaceutical care plan was very effective in recording the pharmacist's ward activities and the pharmaceutical care provided. However, further studies are required in order to explore long-term clinical pharmacists in-put using a well-established electronic care plan; part of Al-Shifa computer system in Omani health centers" [1]. According to Abah et al. "Pharmacotherapy for patients infected with human immunodeficiency virus (HIV) is complex and increases the potential for drug therapy problems (DTPs). We described the frequency and type of DTPs in a Nigerian cohort of HIV infected patients on antiretroviral therapy (ART), as well as the changes in HIV clinical outcomes after pharmacists' intervention.

Pharmacist intervention resulted in clinically significant improvements in patients HIV virological and immunological outcomes. This highlights an important role for the pharmacist in the treatment and care of HIV-infected patients, in a multidisciplinary team" [2] and Colombo stated that "To present a brief overview of human immunodeficiency virus (HIV) infection and to describe the implementation of pharmaceutical care services for adult patients with 
HIV infection. A pharmacist joined a multidisciplinary team serving HIV-infected patients in January 1994.

Current pharmacy services include taking medication histories, educating patients, counseling patients on compliance, monitoring response to therapy, identifying drug-related problems, documenting all interventions, and making therapeutic decisions and formulary choices. The pharmacist also participates in research.

The pharmacist sees patients immediately after their physician appointments. Patients with one or more of the following characteristics are targeted to receive pharmaceutical care: multiple drugs, history of noncompliance, initiation of new drug therapy, recently discharged from hospital or emergency department, identification of potential adverse effects, identification of potential drug-drug interactions, and presence of drug toxicities.

Pharmacists are uniquely qualified to provide a wide range of pharmaceutical care services to HIV-positive patients. Pharmacy interventions should lead to improved outcomes and decreased costs of chronic and very expensive disease" [3]. Carnevale et al. states that "Studies have shown that pharmaceutical care can result in favorable clinical outcomes in human immunodeficiency virus (HIV) infected patients, however, few studies have assessed the economic impact. The objective of this study was to evaluate the clinical and economic impact of pharmaceutical care of HIV-infected patients. A controlled ambispective study was conducted in Brazil from January 2009 to June 2012.

This work demonstrated that pharmaceutical care of HIV-infected patients, for a one-year period, was able to decrease the number of pharmacotherapy problems. However, the clinical outcomes and the costs did not have statistical difference but showed higher percentage of better clinical outcomes and lower costs for some items [4]. Imaura et al. states that "Pharmacists are providing pharmaceutical care in general wards, but it is still not common in the intensive care unit (ICU). However, we have worked in ICU, and recommended the rational dosage regimen of the antibiotics to the physicians during the treatment period. Especially, the patients who were infected with methicillin-resistant Staphylococcus aureus (MRSA) in ICU should be provided appropriate antibiotic therapy; otherwise they have a poor prognosis. Therefore, it was suggested that the pharmacists in the ICU should contribute to optimize the anti-MRSA therapy and reduce the medical cost" [5].

According to Luisetto et al. "Clinical pharmacist presence in many medical team results in general improvement also in some clinical outcomes" [6]. Luisetto et al. also states that "Observing the evolution of clinical pharmacist since today, we are in progressive improvement in clinical outcomes was obtained" [7]. He also states that reduction of medication therapy errors is not only needed but also demanded by both patients and health authorities and government. Multiprofessional healthcare team is the right keyword in today health care systems. A clinical pharmacist today contributes in oncology, toxicology, emergency medicine, nuclear medicine, infectious diseases, nephrology, nutrition service and others. In order to create a team in an efficient fashion, and assess pharmacological therapy related to new biological agents, the pharmacists must go up-to-date their knowledge, so they will be prepared to use the data provided by imaging, medical laboratory, biochemistry, molecular biology, genetics, and immunohistochemistry departments [8]. The knowledge in field of medical laboratory and imaging gives great advantages in this new discipline for the hard relationship with many drug therapies [8]. For this reason also clinical pharmacist must be involved. We strongly ask to public institution to apply this new discipline to obtain more rational drug therapies and rational method to use the clinical pharmacist resource [9]. In some country, it is not clearly accepted the consultant role of clinical pharmacist, in therapy field in improving some clinical outcomes by participation in multidisciplinary medical team [6]. To be part of this team, in efficiently way, there is the need to have a great skill in communications, conflict management, proactivity, resilience, perseverance, critical thinking and others. At the same time, clinical pharmacist (expertise in field of diagnostic science (as medical laboratory and imaging) is not clearly accepted by scientific community (even if diagnostic data are used commonly in monitoring of many drugs therapy). A rapid introduction in medical team is the right keywords [10]. We ask to the international organization involved in healthcare accreditation to introduce a strictly requirement in hospital surgery accreditation: The stabile presence of clinical pharmacist in surgical medical team and to the university institutions to deep introduce this professionalism in core curriculum of clinical pharmacist with topic but also practice applications (surgery rotations). We think that using the classic management instrument added to ICT, professional social media and sharing economy principles we can have an improvement in health care results. By sharing experience, knowledge, skills and information we can have efficient systems more than past [11].

Xiao et al. states that, if a comparative study conducted in a cardiology unit of a university-affiliated hospital. The clinical pharmacists did not perform any intervention associated with improper use of medications during Phase I (pre-intervention) and consulted with the physicians to address the DRPs during Phase II (post-intervention). The primary endpoint of the study was the composite of all-cause mortality during Phase I and Phase II.

DRPs that were suspected to cause or contribute to a possibly fatal outcome were determined by clinical pharmacist service in patients hospitalized in a cardiology ward. Correction of these DRPs by physicians after pharmacist's advice caused a significant decrease in mortality as analyzed by PS matching. The significant reduction in the mortality rate in this patient population observed in this study is "hypothesis generating" for future randomized studies [12].

According to Marston, et al. "The development of antibiotics is considered among the most important advances of modern science. Antibiotics have saved millions of lives. However, antimicrobial resistance (AMR) threatens this progress and presents significant risks to human health. To identify factors associated with AMR, the current epidemiology of important resistant organisms, and possible solutions to the AMR problem. The increase in AMR has been driven by a diverse set of factors, including inappropriate antibiotic prescribing and sales, use of antibiotics outside of the health care sector, and genetic factors intrinsic to bacteria. The problem has been exacerbated by inadequate economic incentives for pharmaceutical development of new antimicrobial agents. Alternative approaches to address the AMR threat include new methods of antibacterial drug identification and strategies that neutralize virulence factors. Antimicrobial resistance poses significant challenges for current clinical care. Modified use of antimicrobial agents and public health interventions, coupled with novel antimicrobial strategies, may help mitigate the effect of multidrug-resistant organisms in the future [13].

Alex, et al. stated that "Our findings have significant repercussions for antibiotic optimisation and streamlining. The increasing selfperception of pharmacy as antibiotic gatekeeper opens up significant 
potential for individual pharmacists to influence antibiotic decisionmaking in everyday practice. However, the resistance of doctors to pharmacy advice and the inconsistencies in pharmacy practice impose severe constraints on the implementation of hospital policies aiming at the optimisation of antibiotic prescribing. These need to be addressed, for example via the design of institutional mechanisms that are sensitive to the differences in professional culture and provide pharmacy with a stronger foothold at the bedside. This includes the reallocation of resources to acknowledge hospital pharmacy's responsibilities beyond the dispensing of medicines and provide them with opportunities to accumulate specialized clinical experience. Increased presence of pharmacists on hospital wards across high resource and low resource settings would also allow the establishment and maintenance of trusting relationships between pharmacists and doctors that promotes a sense of shared obligation to the "patient good" and a mutual understanding as partners in the effort to curb the proliferation of AMR [14]. Brown et al. "The looming antibioticresistance crisis has penetrated the consciousness of clinicians, researchers, policy makers, politicians and the public at large. The evolution and widespread distribution of antibiotic-resistance elements in bacterial pathogens has made diseases that were once easily treatable deadly again. Unfortunately, accompanying the rise in global resistance is a failure in antibacterial drug discovery. Lessons from the history of antibiotic discovery and fresh understanding of antibiotic action and the cell biology of microorganisms have the potential to deliver twenty-first century medicines that are able to control infection in the resistance era" [15].

Candice et al. states "Antimicrobial Therapy critical care clinical pharmacists receive extensive training in infectious disease pharmacotherapy to complement their training in other domains of critical care practice. Moreover, the literature suggests the benefit of clinical pharmacist activity as it pertains to infectious disease pharmacotherapy in critically ill patients. In a single-centered study, the direct contribution from the clinical pharmacist through interpretation of microbiology results, review of patient records, and recommendation of antimicrobial agents was associated with a reduced time to administration of optimal antimicrobial therapy from $64.7 \pm 36.8$ to $39.3 \pm 15.5$ hour $(\mathrm{P}=0.002)$ [10]. Additionally, deescalation of antimicrobial therapy in response to specific culture findings may reduce the emergence of antimicrobial resistance [11]. Guidelines from the Infectious Diseases Society of America and the Society for Healthcare Epidemiology of America state that a clinical pharmacist with infectious disease training should be a core member of the multidisciplinary antimicrobial stewardship team $[12,13]$. This recommendation takes on heightened importance in the ICU, where antimicrobial consumption is greater than in other inpatient care areas.

The impact of the addition of a clinical pharmacist to an antimicrobial control program was evaluated in a Veteran Affairs medical center [14]. Over a 2-year period, the pharmacist reviewed 1,329 orders for restricted and non-formulary antibiotics for appropriateness on the basis of presumed infection, culture results, formulary availability, and dosing. The pharmacist recommended changes in $>50 \%$ of orders, with most changes related to spectrum of activity, formulary substitution, or dose adjustments. These interventions were associated with shorter lengths of stay (10.8 days $v s$. 13.2 days, $\mathrm{P}<0.001)$ and decreased mortality $(6.61 \%$ vs. $8.28 \%$, $\mathrm{P}=0.007)$ compared with the period before the implementation of an antimicrobial control program directed by a clinical pharmacist. A similar impact was seen when pharmacists managed surgical antimicrobial prophylaxis [15].

A survey and database review of 242,704 medicare surgical patients from 806 different hospitals showed that pharmacist-managed antimicrobial prophylaxis was associated with significant improvement in clinical outcomes, such as decreased surgical site infections and length of stay. Nearly $19 \%$ of the hospitals included had pharmacistmanaged surgical prophylaxis. In hospitals that did not offer pharmacist-managed antimicrobial prophylaxis, annual death rates were 52\% higher, with 105 excess deaths (or, 1.54; 95\% CI, 1.4-1.63; $\mathrm{P}<0.0001$ ); length of hospital stay was $10.2 \%$ longer, with 167,941 excess patient days $(\mathrm{P}<0.0001)$; and infection complications were $34.3 \%$ higher (or, 1.52; 95\% CI, 1.40-1.66; $\mathrm{P}<0.0001$ ) than in those with pharmacist involvement. Clinical pharmacists provide expertise in pharmacokinetics and pharmacodynamics essential for dosing considerations in critically ill patients [16-18]. Vancomycin and aminoglycosides are the most common drug therapies managed by a clinical pharmacist. A database review of pharmacist management of vancomycin and aminoglycosides was evaluated in a study population comprising 199,082 Medicare patients treated in 961 hospitals [16]. Hospitals with pharmacist-managed vancomycin or aminoglycoside therapies had lower mortality $(17 \%$ vs. $18 \%, \mathrm{P}<0.0001)$; shorter lengths of stay $(11.56 \pm 18.73$ days vs. $12.98 \pm 18.66$ days, $\mathrm{P}<0.0001)$; and fewer adverse events, including hearing loss $(4.6 \%$ vs. $6.8 \%, \mathrm{P}<0.0001)$ and renal impairment $(25.8 \%$ vs. $34.5 \%, \quad \mathrm{P}<0.0001)$. Similarly, individualized pharmacokinetic monitoring performed by a clinical pharmacist was associated with less aminoglycoside-associated nephrotoxicity compared with physician-monitored therapy in a retrospective case-control study of 2,405 patients $(7.9 \%$ vs. $13.2 \%$, $\mathrm{P}=0.02)[18]$.

The overall effect of a pharmacist on clinical outcomes in patients with known infections was investigated by combining data from the 2006 survey of ICU pharmacy services previously described with International Classification of Diseases, Ninth Revision, Clinical Modification codes and data from the Centers for Medicare \& Medicaid Services [19]. Compared with ICUs with clinical pharmacists, mortality rates in ICUs that did not have clinical pharmacists were higher for nosocomial-acquired infections, community-acquired infections, and sepsis by $23.6 \%$ (386 excess deaths, $\mathrm{P}<0.001$ ), $16.2 \%$ (74 excess deaths, $\mathrm{P}=0.008)$, and $4.8 \%$ (211 excess deaths, $\mathrm{P}=0.008$ ), respectively. Similarly, ICU length of stay was longer for all infection categories by $7.9 \%$ (14,248 excess days, $\mathrm{P}<0.001), 5.9 \%(2,855$ excess days, $\mathrm{P}=0.03)$, and $8.1 \%(19,215$ excess days, $\mathrm{P}<0.001)$, respectively" [16].

According to Oman et al., "In infectious disease ward the clinical pharmacist contribute to resolve about $70 \%$ of drug therapy problem and provided by reviewing patient prescription and counseling discharged patients" [1].

- Abah et al. states that "In HIV therapy pharmacist intervention in a multidisciplinary team resulted clinically significant" [2].

- Colombo et al. states that "The pharmaceutical care service include identify drug related problems, making therapeutic decision, identifying toxicity and potential interactions" [3].

- The pharmaceutical care service in infectious disease setting reduce the costs in very expensive drugs therapy [3].

- Carnevale et al. states that "Pharmaceutical Care service reduce HIV pharmacotherapy problems" [4]. 
- Imaura et al. states that "The pharmacist in ICU contributed antiMRSA therapy and reduces the medical costs" [5].

- Luisetto, et al. observed a progressive improvement in clinical outcomes [6].

- Luisetto, et al. observed the evolution of clinical pharmacist role in medical team [7].

- Clinical pharmacist presence in medical team results in general improvement also in some clinical outcomes [6].

The pharmacist knowledge in field of medical lab and imaging give a great advantages in therapy field because this discipline are heavy involved in drug therapy management [9]. All-cause mortality was $1.5 \%$ during Phase I (pre-intervention) and was reduced to $0.9 \%$ during Phase II (post-intervention), and the difference was statistically significant $(\mathrm{P}=0.0005)$. After PS matching, all-cause mortality changed from $1.7 \%$ during Phase I down to $1.0 \%$ during Phase II, and the difference was also statistically significant $(\mathrm{P}=0.0074)$ [12]. According to Al-Somai et al. "This study showed improvement in antimicrobial use after the intervention of the clinical pharmacist and ID. Involvement of the clinical pharmacist and ID is highly recommended to optimize the use of antimicrobials, and decrease antimicrobial resistance in hospitalized patients. We recommend continuation of the study to maintain supervision over quality, decrease resistance, and monitor the need for intervention in the different wards. Surveillance of consumption of reserved antibiotics should be done according to local guidelines, as well as for external comparison. This can save medical staff the burden of managing patients chronically, or having deteriorating patients in the wards [17]. Bessesen, et al. states that "An antimicrobial stewardship program with a dedicated ID pharmacist was associated with greater adherence to recommended antimicrobial therapy practices when compared to a stewardship program that relied on ward pharmacists [18]. In a lancet infection disease meta-analysis was reported. Our findings of beneficial effects on outcomes with nine antimicrobial stewardship objectives suggest they can guide stewardship teams in their efforts to improve the quality of antibiotic use in hospitals [19].

\section{Discussion}

Analyzing the article reported in this paper we can see a relevant role of clinical pharmacist in field of infectious disease. Infectious clinical pharmacist provide direct patient care in various inpatient settings (internal medicine a, critical care hemology/oncology, solid organ transplant with other, as well as outpatient settings such as HIV clinics). Antibiotic stewardship and surveillance programs can be considered efficacy tools available today as well as a guideline of protocols, procedure, EBM criteria and many others. Clinical pharmacokinetic consultant service, microbiologic and laboratory assay and assessment, scientific drug information, toxicity management, $\mathrm{ADR}$, interactions, medicinal chemistry competences are core curriculum of clinical pharmacist course and for this reason the permanent presence of clinical pharmacists results in general positive outcome in many clinical team [6].

\section{Conclusion}

The antimicrobial therapy managed also by clinical pharmacist competences today more than past gives general improvement in clinical and economic outcomes in

- $\quad$ Severe disease (as Hepatitis C and others).
- Control of some pathology as HIV and others with mortality reduction.

- Reducing or controlling MDR diffusion.

- Reducing healthcare associated cost.

- Multi-disciplinary decision making system surely contribute to the final endpoint.

A new discipline named Clinical pharmaceutical care can be the right instruments also in infectious disease medical team and a rapid introduction in medical team by clinical pharmacist Give improving in clinical and economic outcomes and in antimicrobials global management [10].

\section{Declaration}

This paper is not for any diagnostic or therapeutic intent but focused for clinical pharmacist use to have a general view and to have a rapid introduction in infectious disease medical team and instruments to deep apply in the systematic infectious disease management.

\section{References}

1. Al Salmi Z (2009) Clinical audit of pharmaceutical care provided by a clinical pharmacist in cardiology and infectious disease in-patients at the Royal hospital, Muscat/Oman. Oman Med J 24: 89-94.

2. Abah IO, Ojeh VB, Falang KD, Darin KM, Olaitan OO, et al. (2014) Pharmaceutical care outcomes in an outpatient human immunodeficiency virus treatment center in Jos, Nigeria. J Basic Clin Pharm 5: 57-61.

3. Colombo J (1997) Establishing pharmaceutical care services in an HIV clinic. Am Pharm Assoc (Wash) 37: 581-592.

4. Carnevale RC, Rezende CG, Molino C, Visacri MB, Mazzola PG, et al. (2015) Cost analysis of pharmaceutical care provided to HIV-infected patients: An ambispective controlled study. Daru 23: 13.

5. Imaura $\mathrm{M}$, Kohata $\mathrm{Y}$, Kobayashi $\mathrm{K}$, Takahashi $\mathrm{H}$, Yokoyama $\mathrm{H}$, et al. (2011) Effect of pharmacists' intervention on the antibiotic therapy for the methicillin-resistant Staphylococcus aureus (MRSA) infectious diseases in the intensive care unit. Yakugaku Zasshi 131: 563-570.

6. Luisetto M, Carini F, Bologna G, Nili-Ahmadabadi B (2015) Pharmacist cognitive service and pharmaceutical care today and tomorrow outlook. UKJPB 3: 67-72.

7. Luisetto M, Nili-Ahmadabadi B, Cabianca L, Ibne M (2016) Steps and impacts of pharmaceutical care and clinical pharmacy development on clinical outcomes. A historical analysis compared with results. Clinicians Teamwork bulletin 1: 4-8.

8. Luisetto M, Nili-Ahmadabadi B (2016) An Open Letter to all Pharmacists: Pharmaceutical Care, Medical Laboratory and Imaging 2016 Clinician teamwork bulletin 1: 1-3.

9. Luisetto M, Sahu RK (2016) Clinical Pharmaceutical Care: A new management health care discipline in 2016. UK J Pharm \& Biosc 4: 63-64.

10. Luisetto M (2016) Psychological and Behavior skills for Ph. care practice in medical team 2016, IJPPR 5: 1-4

11. Luisetto M (2016) Pharmaceutical Care in Surgery Field 2016. J Pharma Care Health Sys 3: 3 .

12. Zhai X, Gu Z, Liu X (2016) Effectiveness of the clinical pharmacist in reducing mortality in hospitalized cardiac patients: A propensity scorematched analysis. Ther Clin Risk Manag 12: 241-250.

13. Marston HD, Dixon DM, Knisely JM, Palmore TN, Fauci AS (2016) Antimicrobial Resistance. JAMA 316: 1193-1204.

14. Broom A, Plage S, Broom J, Kirby E, Adams J (2015) A qualitative study of hospital pharmacists and antibiotic governance: Negotiating interprofessional responsibilities, expertise and resource constraints. BMC Health Serv Res 16: 43. 
Citation: Luisetto M (2017) Infectious Disease Pharmaceutical Care: The Role of the Clinical Pharmacists to Improve Clinical Outcomes 2017. J Antimicrob Agents 3: 143. doi:10.4172/2472-1212.1000143

Page 11 of 11

15. Brown ED, Wright GD (2016) Antibacterial drug discovery in the resistance era. Review Nature 529: 336-343.

16. Preslaski CR, Ishaq L, MacLaren R, Poston J (2014) Pharmacist Contributions as Members of the Multidisciplinary ICU Team. Chest 144 1687-1695.

17. Al-Somai N, Al-Muhur M, Quteimat O, Hamzaha N (2014) The impact of clinical pharmacist and ID intervention in rationalization of antimicrobial use. Saudi Pharm J 22: 516-521.
18. Bessesen MT, Ma A, Clegg D, Fugit RV, Pepe A, et al. (2015) Antimicrobial stewardship programs: Comparison of a program with infectious diseases pharmacist support to a program with a geographic pharmacist staffing model. Hosp Pharm 50: 477-483.

19. Schuts EC, Hulscher ME, Mouton JW, Verduin CM, Stuart JW, et al. (2016) Current evidence on hospital antimicrobial stewardship objectives: A systematic review and meta-analysis. Lancet Infect Dis 16: 847-856. 